\title{
Ultrastructure of the Ovarian Germ Cells in the Quail Embryos, with Special Reference to the Oocytes
}

\author{
By \\ Atsumi UKESHIMA \\ Department of Anatomy and Pathology, School of Health Sciences, Kumamoto University, \\ Kumamoto 862-0976, Japan \\ - Received for Publication, October 16, 2003 -
}

\begin{abstract}
Key Words: Oogonia, Oocytes, Quail, Germ cells, Ultrastructure
\end{abstract}
\begin{abstract}
Summary: Developing germ cells from oogonia to oocyte stages in embryonic quail ovaries were observed ultrastructurally. In contrast with those of chicks, quail oocytes showed some conspicuous structures. Typical feature of the Balbiani body, which was constructed by eccentrical concentration of whole organelles, was observed through the oocyte stage, although loose aggregations of organelles were found even at the oogonial stage. Such striking feature of Balbiani body was not observed as yet in any animal species. Golgi apparatus, which occupied the center of a Balbiani body, was often found attaching with the nuclear membrane. This finding suggests that the Balbiani body play some role for the oocyte maturation.

Upon the nuclear membrane of oocytes, a great number of electron dense structures were found. They were thought to be a kind of nuclear pore complexes. Such abundance of the nuclear pore complexes was another structural characteristic of quail oocytes. They increased in number according to the progress of embryonic development, although small numbers were also seen even at the oogonial stage.
\end{abstract}

In embryonic development of avian species, multiplication of germ cells (oogonia) proceeds within the cortex being in only the left ovary, after their arrival from extragonadally original region. Consequently, the left ovarian cortex becomes occupied with many clusters or masses of proliferated oogonia.

A series of our investigations on the germ cells of chick embryos, from primordial germ cells to oocytes, have demonstrated several fine morphological characteristics (Fujimoto et al., 1976; Ukeshima and Fujimoto, 1984; Ukeshima and Fujimoto, 1991; Fujimoto and Ukeshima, 1997). Particularly, fragmented nucleolus was the most conspicuous characteristic of chick germ cells. From 9 days of incubation onwards, the chick germ cells in the left ovary increased their number as oogonia. Then, the earliest oogonia developed into oocytes at about 12 days, showing meiotic feature. Almost all ones entered meiosis by 15 days. During those periods, chick oocytes exhibited a conspicuous appearance. In the cytoplasm, so-called "Balbiani body" (Greenfield, 1966; Guraya, 1979; yamada and Amanuma, 1980), which was made of concentrated organelles, was appeared.

In the case of quails, on the other hand, the germ cells showed somewhat different profile from the chicks at fine structural level (Yoshinaga et al., 1993). However, details are unknown yet, particularly in later period of the development. In the present study, therefore, we aimed to clarify the fine structural characteristics of quail germ cells from oogonia to oocyte stages.

\section{Materials and Methods}

As well as in other avian species, quail ovary developed only on the left side. Therefore, left ovaries from 9 to 15 day embryos were used for the present study. The left ovaries excised from the embryos were fixed with $2.5 \%$ glutaraldehyde (0.1 M cacodylate buffer $\mathrm{pH} 7.4$ ) for $1.5 \mathrm{hr}$ under cool condition. Then, materials were post-fixed with $1 \%$ osmic acid (0.1 M cacodylate buffer $\mathrm{pH} 7.4$ ) for $1 \mathrm{hr}$ under same condition. After washing in same buffer, materials were dehydrated in graded series of ethanol and embedded in epoxy resin. All sections including thick and ultrathin sections were made transversely. Thick sections were stained with toluidine blue for light microscopy. While, ultrathin sections were stained with uranyl acetate and lead citrate before the observation with electron microscopy. 


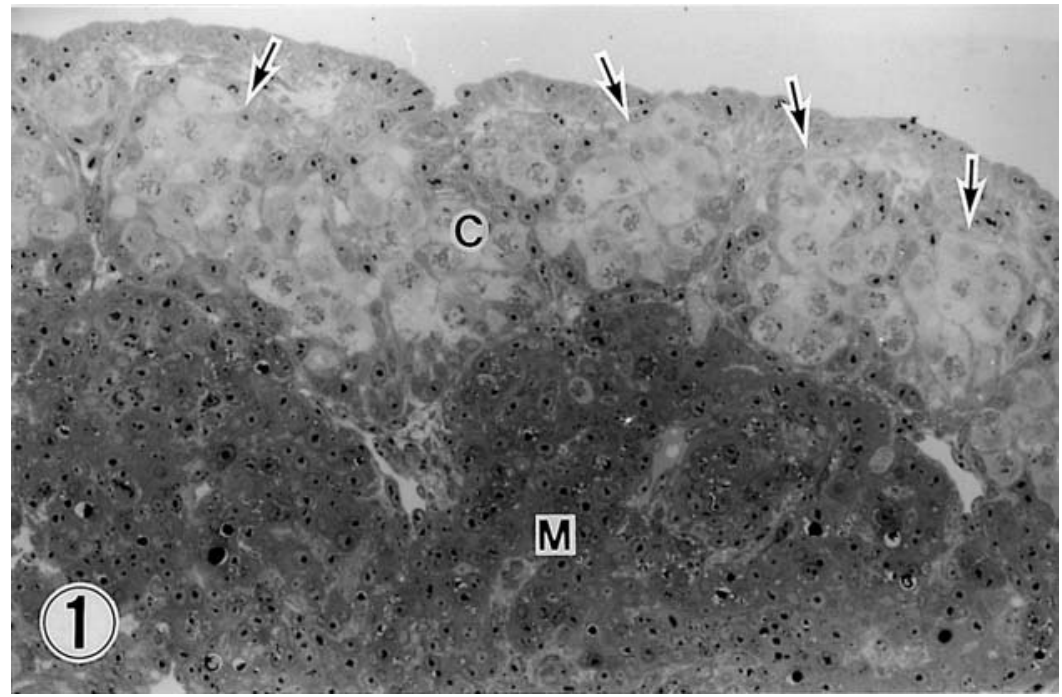

Fig. 1. Light micrography of a cross section of the left ovary from 13 day-embryo. The cortex $(\mathrm{C})$ appears light tone compared with the medulla (M). Proliferated germ cells (oocytes) make many germ cell clusters, or masses (arrows) in the cortex. In each oocyte, a nucleus and Balbiani body can be recognized more or less darkly. $\times 320$

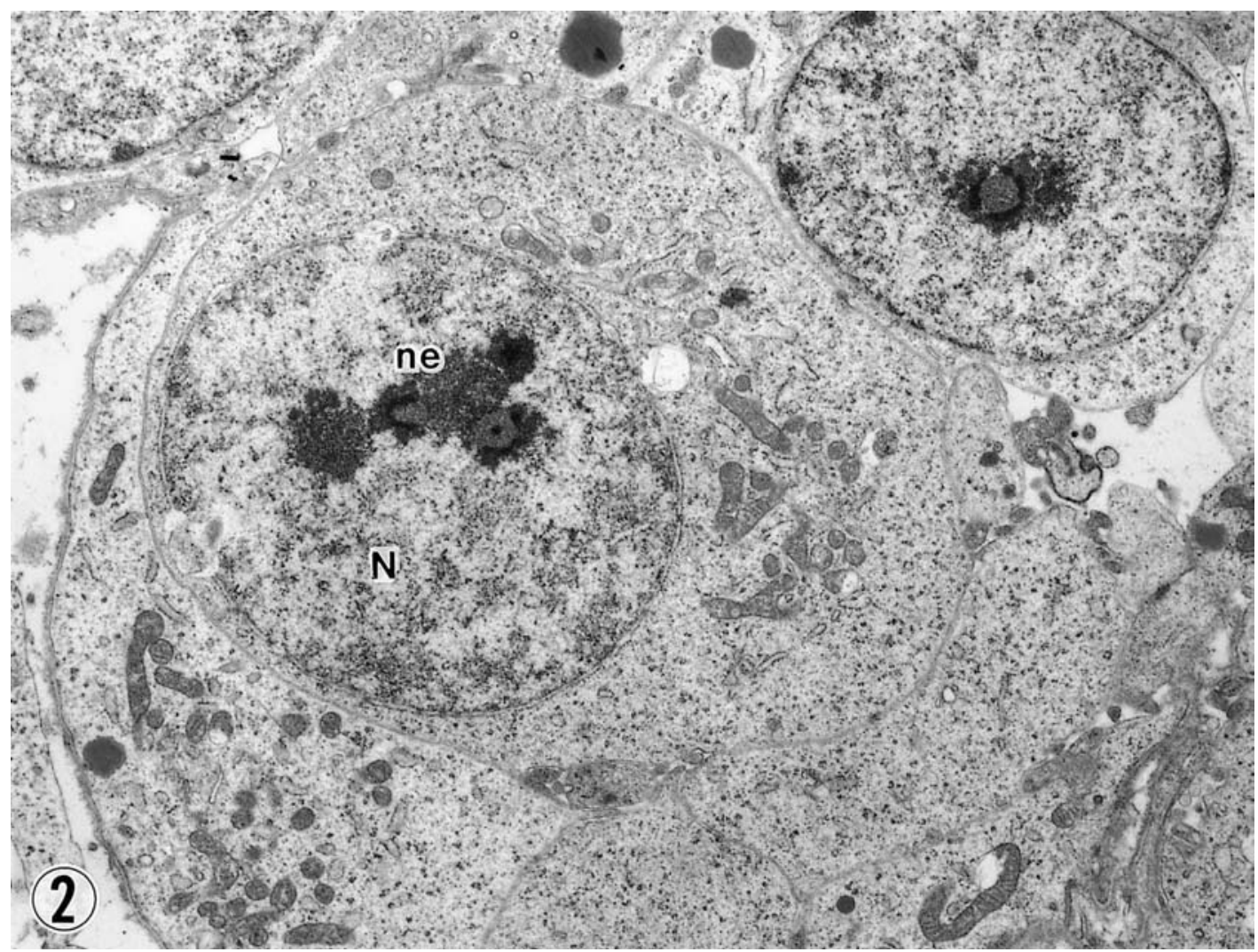

Fig. 2. Oogonium found in the cortex of 9 day left ovary. The nucleus (N) shows spherical contour. The nucleolus (ne) is composed of granular mass and highly dense fine particles. Organelles take loosely one-sided position within the ooplasm. $\times 8,700$ 


\section{Results}

\section{Light microscopy}

The left ovaries of the quail embryos at 9-10 days of incubation were composed of two areas, i.e., cortex and medulla. The cortex was a functional layer of a female gonad, and majority of germ cells were included in this layer. While, in the medulla germ cells were rarely found. At this period, germ cells called oogonia were seen to proliferate to make germ cell masses.

At 13 days, the cortex was seen thickened increasingly by oogonial proliferation, measuring about 7 to $10 \mu \mathrm{m}$ in thick, although it varied depending on a sectioned level in the ovary. The cortex was seen apparently light tone compared to the medulla. In the cortex, oogonia increased in number with the advance of embryonic development, and formed many large cell masses or clusters by aggregation of proliferated cells. At this period, majority of the oogonia developed to oocytes, and entered meiotic division.

Higher magnification showed detailed profiles
(Fig. 1). Each oocyte possessed a nucleus including the forming chromosomes by meiosis and a Balbiani body which was the aggregation of organelles, and was seen paler than the neighboring somatic cells.

\section{Ultrastructure of the oogonia/oocytes}

Thickened cortex of the left ovary contained many clusters composed of proliferated oogonia. An oogonium had a large, spherical nucleus, which usually took an eccentric position of the cell and contained one or two nucleoli (Fig. 2). The nucleus included fine particles, and lacked the heterochromatin aggregation as seen in somatic cells, usually exhibiting light tone as a whole. The nucleolus showed an irregular contour, composing of dense granulated masses and extremely dense materials attached those. Organelles were usually scattered in the cytoplasm, but sometimes seen getting together in some degree at one side of the cell. Free ribosomes were distributed throughout the cytoplasm, usually as polysomes.

At 12 days, many of the oogonia had already

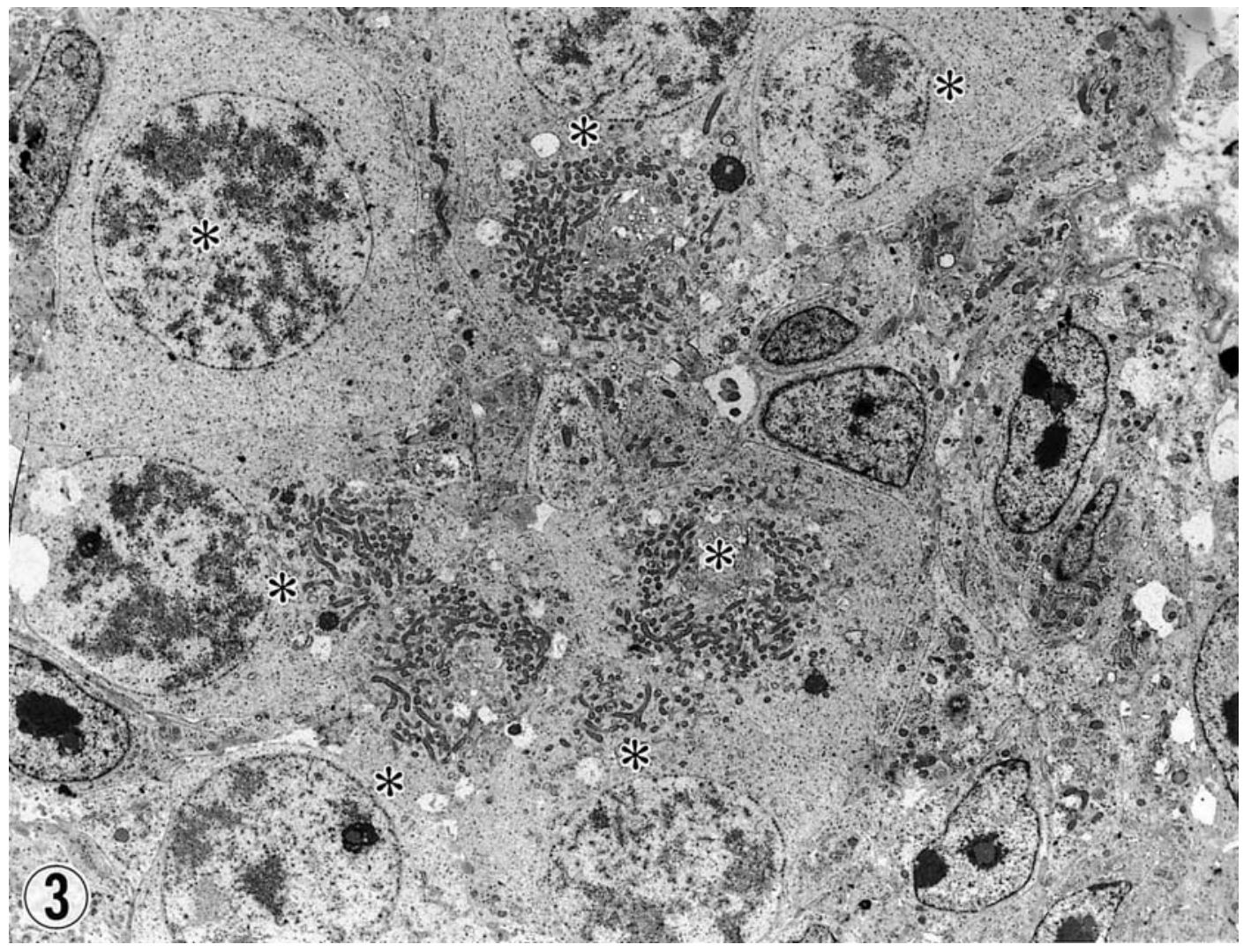

Fig. 3. A Low magnification figure of a germ cell cluster including many oocytes (asterisks) in the cortex from the 13-day ovary. Each oocyte shows meiotic prophase. As cell organelles concentrate tightly to make the Balbiani body, the oocyte is seen to have two cores formed by nucleus and Balbiani body. $\times 3,400$ 


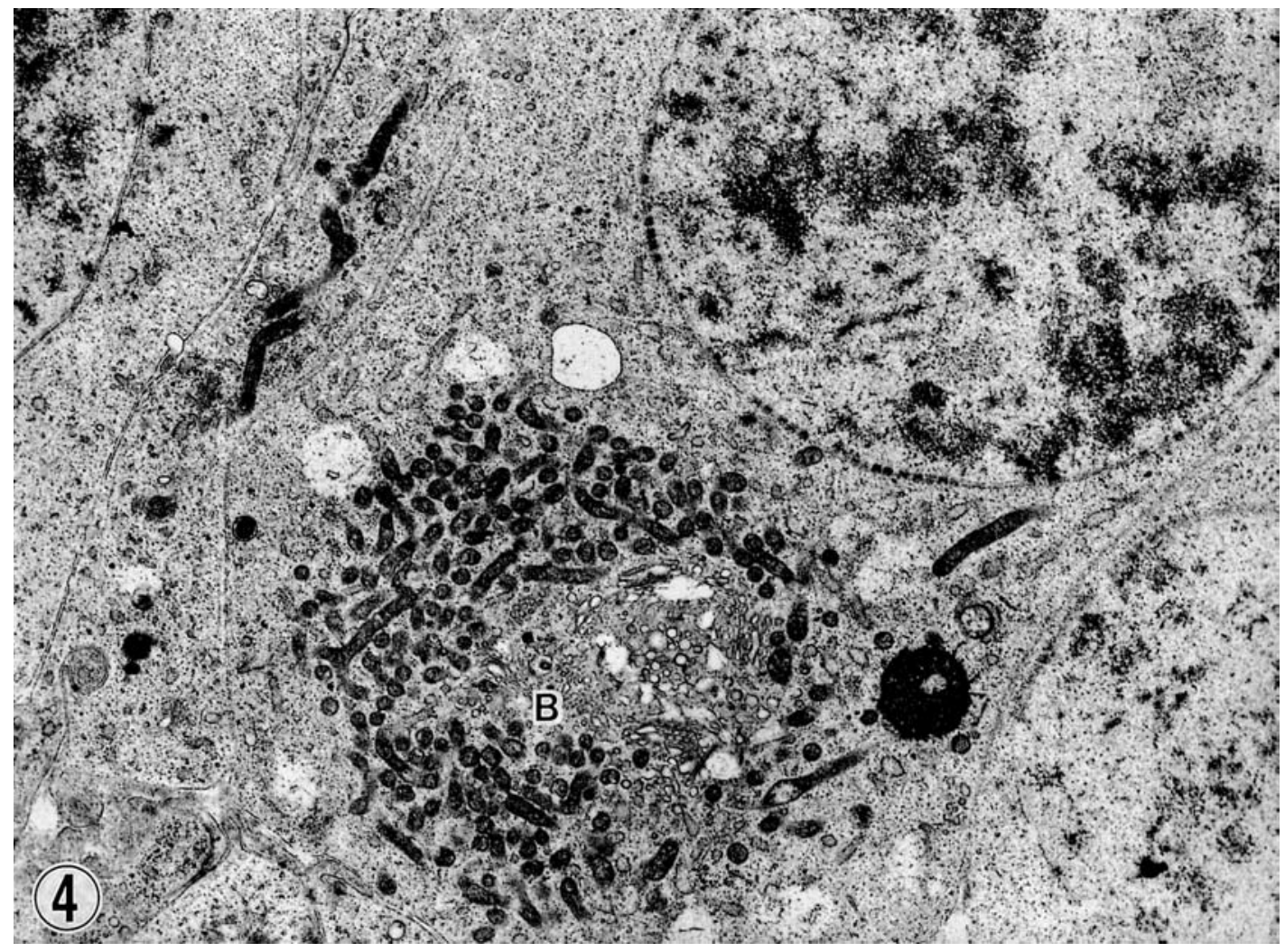

Fig. 4. High magnification figure of a oocyte from 13-day ovaries. Almost all organelles are tightly concentrated to form Balbiani body. In the Balbiani body (B), numerous mitochondria surround the Golgi complex. Peripherally, some vacuoles and electron dense body are seen. $\times 10,000$

developed to oocytes, showing miotic feature. Although progress of the meiotic division seemed to vary depending on cells, many oocytes exhibited a zygotene stage. From 13 days onwards, oogonia were seldom found. Synaptinemal complex attaching to the nuclear membrane of the oocytes was clearly recognized.

In the oocytes, all organelles were closely concentrated near the nucleus, making a large organelles mass, so-called "Balbiani body" (Figs. 3, 4). The size of the Balbiani body measured about 7 to $8 \mu \mathrm{m}$ in diameter. At lower magnification, therefore, the cell body of oocytes looked as having two large conspicuous cores, i.e., nucleus and Balbiani body (Fig. 3).

Although a Balbiani body showed various profiles according to the direction of its cut surface, the most typical one was a concentric distribution of the organelles. The central region was occupied with a well-developed Golgi complex, and great numbers of mitochondria had surrounded it. At the periphery, a large and round electron dense body measuring about $1 \mu \mathrm{m}$ in diameter and several vacuoles were present. The Balbiani body was fre- quently found to relate with the nucleus. In such case, Golgi complex was attached to the nuclear membrane (Fig. 5).

Upon the nuclear membrane of oocytes, many electron dense structures, which were thought to be nuclear pore complexes, were found (Figs. 4, 5, 6). Although these structures were seen in small number at the oogonial stage, they became prominently increased with advancing embryo age. Particularly in 15-day embryos, as for that number more than eighty were counted in one section of a nuclear envelope. Their distribution was at random upon a nuclear membrane, closely or very sparsely. Generally, the density of their distribution was very high in later developmental stages. At the most closely distributed area, it was about $50 \mathrm{~nm}$ in distance.

\section{Discussion}

In avian embryos, primordial germ cells initially originate in an extra-embryonic area, and then migrate into the developing gonads (Fujimoto et al., 


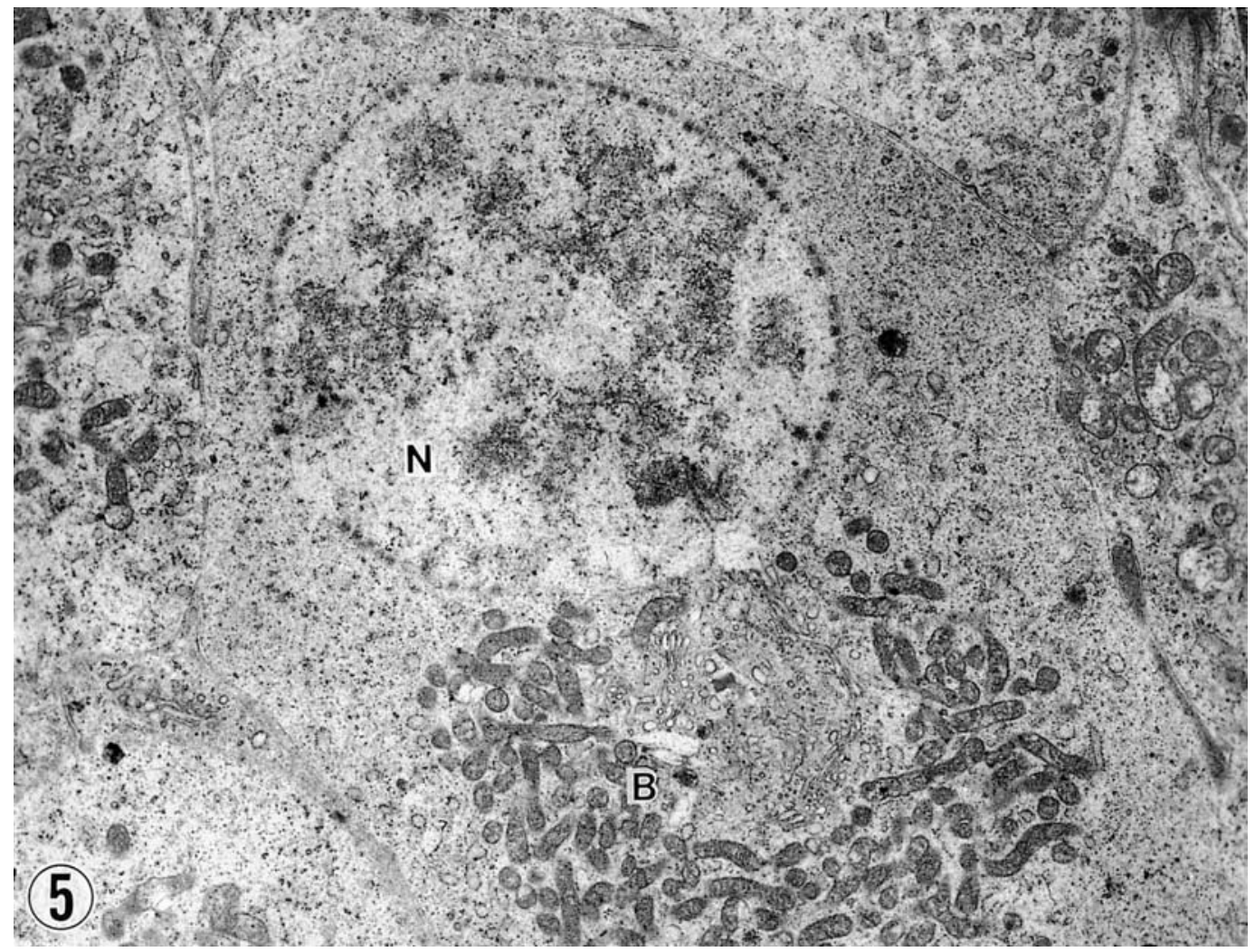

Fig. 5. This figure shows the Balbiani body (B) in contact with the nucleus (N) from a 15-day oocyte. Golgi complex located in a center of the Balbiani body is attached to the nuclear membrane. Upon the nuclear membrane, many electron dense structures considered as nuclear pore complexes are seen. Their distribution is of unequal. $\times 10,000$

1976; Fujimoto and Ukeshima, 1984). Thereafter, germ cells proliferate and differentiate according to the sex of the embryo. In females, germ cells multiply as "oogonia" in the cortex, which is formed only in the left ovary, while the right ovary is to degenerate. This phenomenon is owing to the fact that no cortex is formed in the right ovary. For this reason, ovaries were prepared only the left side as materials of the present study.

According to changing from oogonia to oocytes, they enter the meiotic division. We have observed the ultrastructural characteristics of germ cells in this period by using the chick (Ukeshima et al., 1991; Ukeshima, 1994; Fujimoto et al., 1997), and clarified that the nucleolus of chick germ cells exhibited a fragmented shape even at the oogonial stage. Quail germ cells in early developmental stages, on the other hand, were also investigated fine morphologically. Yoshinaga et al. (1993) observed the quail primordial germ cells, and showed that the quail nucleus had a nucleolus made of one or two dense masses including granular structure, unlike that of the chick. In the present study, it was showed that such a characteristic of a quail nucleus was identical even at the oogonial stage.

In oocytes cytoplasm, almost all organelles concentrated to make a so-called "Balbiani body" (Yamada and Amanuma, 1980). The Balbiani body was described in many animal species and also called 'yolk nucleus' (Greenfield, 1966; Guraya, 1979; Yamada and Amanuma, 1980; Kobayashi and Iwamatsu, 2000). In the present study, the Balbiani body has showed a typical pattern of organelles aggregation, in which a great numbers of mitochondria concentrically surrounded a Golgi complex. Such Balbiani body with predominantly concentrated organelles has not been shown as yet in other animal species. In addition, it was found that Balbiani body was in contact with the nuclear membrane. Balbiani body has been thought to play a role in yolk formation, although it currently is unacceptable. However, this finding in the present investigation may suggest that a Balbiani body play some kinds of role in oocyte maturation. While, at the oogonial stage, loosely gathered organelles area was observed. In such case, those were localized widely at one side of the cell, but small numbers were still seen in other area. It may be thought that 


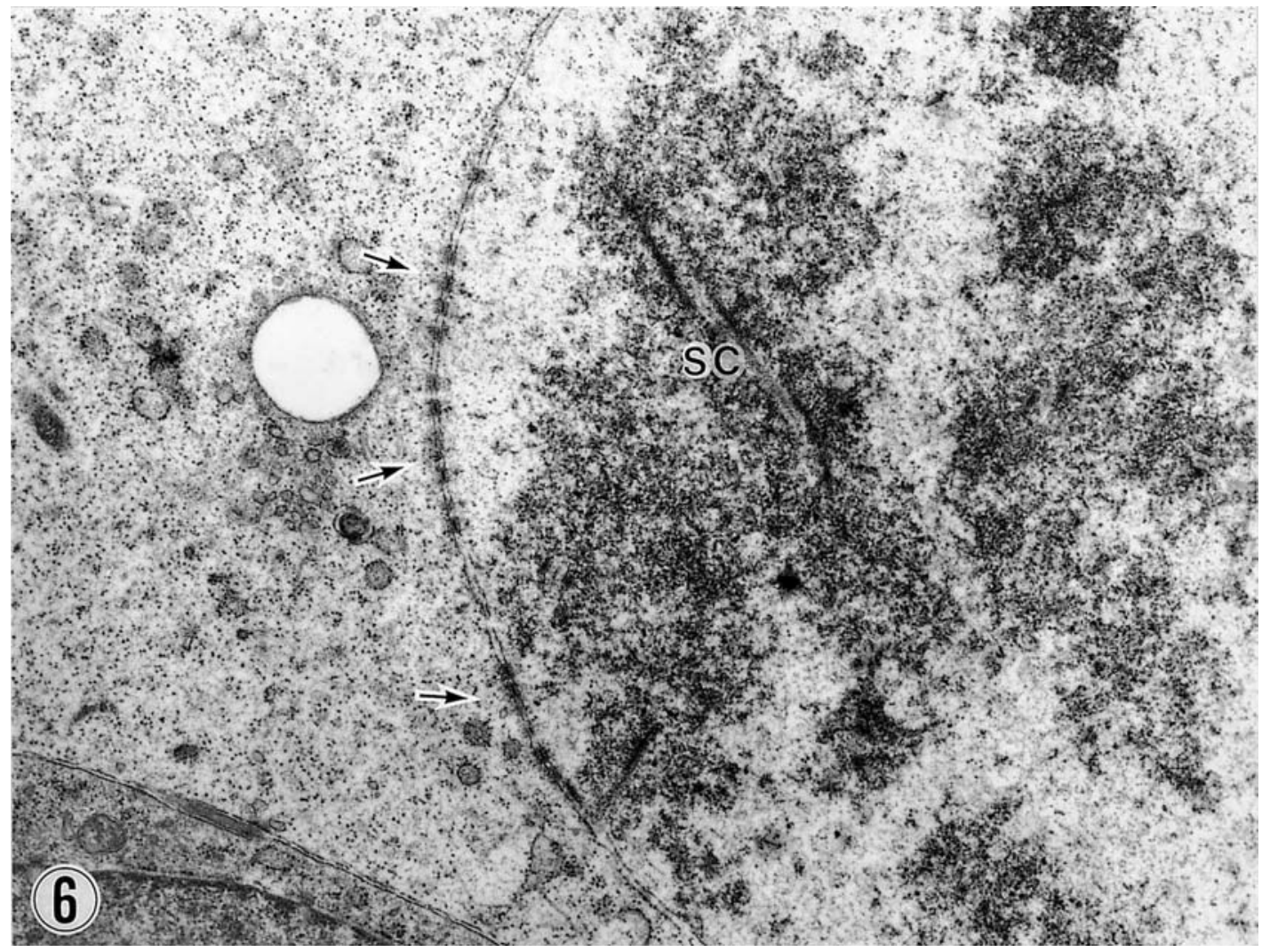

Fig. 6. High magnification figure of a part of the nucleus from a 13-day oocyte. Upon the nuclear membrane, many nuclear pore complexes (arrows) are seen to concentrate. Within the nucleus, synaptinemal complex (SC) is recognized. $\times 17,500$

this phenomenon demonstrates a process to form a Balbiani body.

Upon the nuclear membrane of oocytes, many electron dense structures were observed. They were thought to be a kind of nuclear pore complex, at lower magnification being recognized as electron dense dots. They increased in number with the advance of embryonic development. Particularly, 15day oocytes have extremely large numbers of pores. In the chick oocytes, the presence of such many pores was not described as yet. Therefore, the abundance of pores is thought another characteristic of quail oocytes. The details of this structure, however, remain obscure in quails.

Ovaries used for the present study were taken from 9 to 15-day embryos. In this period, germ cells were seldom found in the medulla. Therefore, the medulla was not used for the materials. They can, however, be found, if embryos earlier than 9 days are employed, because in the chicks germ cells were found also within the medulla of early embryos.

\section{References}

1) Fujimoto T, Ukeshima A and Kiyofuji R. The origin, migration and morphology of the primordial germ cells in the chick embryo. Anat Rec 1976; 185:139-154.

2) Fujimoto $T$ and Ukeshima A. Ultrastructural dynamics of primordial germ cells and gonadal development Comparative aspects - . in Microscopy of reproduction and development: A dynamic approach. Antonio Delfino Editore, Rome, 1997; pp 323-330.

3) Fujimoto $T$ and Ukeshima A. Ultrastructure of primordial germ cells in the early chick embryo. in Ultrastructure of Reproduction. (eds) JV Blerkom and PM Motta, Martinus Nijhoff Publishers, Boston, 1984; pp 12-18.

4) Greenfield ML. The oocyte of the domestic chicken shortly after hatching, studied by electron microscopy. J Embryol exp Morph 1966; 15:297-316.

5) Guraya SS. Recent advances in the morphology, cytochemistry, and function of Balbiani's vitelline body in animal oocytes. International review of cytology. 1979; 59:249-321.

6) Kobayashi $\mathrm{H}$ and Iwamatsu $\mathrm{T}$. Development and fine structure of the yolk nucleus of previtellogenic oocytes in the medaka oryzias latipes. Develop Growth Differ 2000; 42:623-631.

7) Ukeshima A and Fujimoto T. A fine morphological study of germ cells in asymmetrically developing right and left ovaries of the chick. Anat Rec 1991; 230:378-386. 
8) Ukeshima A. Abandonment of germ cells in the embryonic chick ovary: TEM and SEM studies. Anat Rec 1994; 240:261-266.

9) Yamada K and Amanuma A. Fine structure of Balbiani body in germ cells of the chick embryo (in Japanese). J
Predental Faculty Gifi College Dentistry 1980; 6:87-103.

10) Yoshinaga $K$, Nakamura $M$ and Ukeshima A. Ultrastructural characteristics of primordial germ cells in the quail embryo. Anat Rec 1993; 236:547-552. 
